\title{
ASL-BIDS, the brain imaging data structure extension for arterial spin labeling
}

Patricia Clement ${ }^{1^{*}}$, Marco Castellaro ${ }^{2}$, Thomas W. Okell ${ }^{3}$, David L. Thomas ${ }^{4,5}$, Pieter Vandemaele $^{1}$, Sara Elgayar ${ }^{6}$, Aaron Oliver-Taylor ${ }^{7}$, Thomas Kirk ${ }^{8}$, Joseph G. Woods ${ }^{9}$, Sjoerd $\operatorname{Vos}^{10,5}$, Joost P.A. Kuijer ${ }^{11}$, Eric Achten ${ }^{1}$, Matthias J.P. van Osch ${ }^{12}$, the BIDS maintainers ${ }^{13}$, John Detre ${ }^{14,15}$, Hanzhang Lu ${ }^{16}$, David C. Alsop ${ }^{17}$, Michael A. Chappell ${ }^{3,18,19,20}$, Luis HernandezGarcia $^{21}$, Jan Petr ${ }^{11,22}$, Henk Mutsaerts ${ }^{1,11}$

1. Ghent Institute for Functional and Metabolic Imaging (GlfMI), Ghent University, Ghent, Belgium;

2. Department of Neuroscience, Movement and Biomedicine, University of Verona, Verona, Italy

3. Wellcome Centre for Integrative Neuroimaging, FMRIB Division, Nuffield Department of Clinical Neurosciences, University of Oxford, United Kingdom;

4. Dementia Research Centre, UCL Queen Square Institute of Neurology, University College London, Queen Square, London, United Kingdom;

5. Neuroradiological Academic Unit, Department of Brain Repair and Rehabilitation, UCL Queen Square Institute of Neurology, University College London, Queen Square, London, United Kingdom;

6. Faculty of computers and information science, Ain Shams University, Cairo, Egypt;

7. Gold Standard Phantoms, London, United Kingdom;

8. Institute of Biomedical Engineering, Department of Engineering Science, University of Oxford, Oxford, United Kingdom;

9. Center for Functional Magnetic Resonance Imaging, Department of Radiology, University of California San Diego, La Jolla, California, United States;

10. Centre for Medical Image Computing, University College London, London, United Kingdom;

11. Department of Radiology and Nuclear Medicine, Amsterdam University Medical Center, Amsterdam Neuroscience, Amsterdam, The Netherlands;

12. C.J. Gorter Center for high field MRI, Department of Radiology, Leiden University Medical Center, Leiden, The Netherlands;

13. https://bids.neuroimaging.io/governance.html\#bids-maintainers-group

14. Department of Radiology, University of Pennsylvania, Philadelphia, PA, United States;

15. Department of Neurology, University of Pennsylvania, Philadelphia, PA, United States;

16. Department of Radiology, Johns Hopkins University School of Medicine, Baltimore, Maryland, United States;

17. Department of Radiology, Beth Israel Deaconess Medical Center, Harvard Medical School, Boston, Massachusetts, United States;

18. Radiological Sciences, Mental Health and Clinical Neurosciences, School of Medicine, University of Nottingham, Nottingham, UK

19. Sir Peter Mansfield Imaging Center, School of Medicine, University of Nottingham, Nottingham, UK

20. Nottingham Biomedical Research Centre, Queens Medical Centre, University of Nottingham, Nottingham, UK.

21. Functional MRI Laboratory, University of Michigan, Ann Arbor, Michigan, USA;

22. Helmholtz-Zentrum Dresden-Rossendorf, Institute of Radiopharmaceutical Cancer Research, Dresden, Germany.

\section{${ }^{*}$ Correspondence:}

Patricia Clement

patricia.clement@ugent.be 


\begin{abstract}
Arterial spin labeling (ASL) is a non-invasive MRI technique, allowing quantitative measurement of cerebral perfusion. Incomplete or inaccurate reporting of acquisition parameters complicates quantification, analysis, and sharing of ASL data, particularly for studies across multiple sites, platforms, and ASL methods. Therefore, there is a strong need for standardization of ASL data storage, including acquisition metadata. Recently ASL-BIDS, the BIDS extension for ASL, was developed and released in BIDS 1.5.0. This manuscript provides an overview of the development and design choices of this first ASL-BIDS extension, which is mainly aimed at clinical ASL applications. The structure of the ASL data, focusing on storage order of the ASL time series and implementation of calibration approaches, unit scaling, ASL-related BIDS fields, and storage of the labeling plane information, are discussed. Additionally, an overview of ASL-BIDS compatible conversion and ASL analysis software and ASL example datasets in BIDS format is provided. It is anticipated that large-scale adoption of ASL-BIDS will improve the reproducibility of ASL research.
\end{abstract}




\section{Introduction}

Arterial spin labeling (ASL) is a non-invasive MRI technique for the quantitative measurement of perfusion, the most common measure of cerebral blood flow (CBF). Important advances in labeling strategies and readout techniques have resulted in sufficient quality for research and clinical use of ASL in the last decade. The 2015 ISMRM Perfusion Study Group ASL recommendations ${ }^{1}$ have led to the implementation of $3 D$ pseudo-continuous ASL (PCASL) as product sequence by GE, Philips, and Siemens, which improved the comparison of ASL data between multiple centers and studies. Prior multi-vendor reproducibility studies have shown that ASL CBF values are generally comparable across MRI scanner platforms ${ }^{2}$, particularly when the same labeling scheme is used $^{3}$. Nevertheless, acquisition parameters and data management approaches vary across ASL implementations. As accurate ASL image processing and quantification depend on the exact knowledge of the acquisition parameters ${ }^{1,4}$, harmonization and standardization of the data structure are potentially significant steps to guarantee comparable quantification, allowing the pooling of ASL data.

Comparability of datasets and data sharing is often cumbersome due to inconsistent data management procedures and data formats. MR imaging data are heterogeneously organized; each MRI vendor, researcher, and sometimes even each study uses their ad-hoc structure, which sometimes only contains subsets of the parameters required for image processing. This heterogeneity in data organization, description, and storage complicates the combination of ASL data sets from different sites in multi-center studies, requires additional efforts such as manual input of metadata to perform secondary analyses, and complicates automatic data validation, quality control, image processing, and analysis. Unlike contrast-enhanced perfusion MRI, the Digital Imaging and Communications in Medicine (DICOM) standard ${ }^{5}$ is usually not used as the format for processed ASL data. While some ASL-specific DICOM fields are defined, they are incomplete, not mandatory, and rarely used by the vendors ${ }^{6,7}$. Instead, most investigators analyze ASL data in $\mathrm{NIfTI}^{8,9}$ format.

The Brain Imaging Data Structure (BIDS) ${ }^{10}$ - https://bids.neuroimaging.io - proposed in 2016, is a data storage standard, meeting the need for a structured manner to organize imaging data, offers a suitable structure to standardize ASL data. The initial BIDS proposal covered anatomical, functional, and diffusion MRI. Subsequently, extensions for magnetoencephalography $(M E G)^{11}$, electroencephalography $(E E G)^{12}$, intracranial EEG $(\mathrm{iEEG})^{13}$, and Positron Emission Tomography ${ }^{14}$ have been incorporated and several other extension proposals are in development.

In this manuscript, an overview of the development and design choices of the first release of ASL-BIDS is provided. For this first release, only ASL approaches described in the 2015 ISMRM Perfusion Study Group ASL recommendations are included ${ }^{1}$. For the remainder of this manuscript, the reader is assumed to be familiar with ASL terminology, detailed in the 2015 ISMRM Perfusion Study Group ASL recommendations ${ }^{1}$. 


\section{ASL-BIDS specification}

\section{ASL approaches included in this first release}

The existing efforts for standardization, National Electrical Manufacturers Association (NEMA) DICOM C.8.13.5.14 MR Arterial Spin Labeling Macro ${ }^{6,7}$ and the 2015 ISMRM Perfusion Study Group ASL recommendations ${ }^{1}$ were used as the basis for ASL-BIDS. This extension only supports ASL approaches that were recommended or discussed in the 2015 ISMRM Perfusion Study Group ASL recommendations to facilitate adoption: pulsed (PASL) and (pseudo)continuous $((\mathrm{P}) \mathrm{CASL})^{1}$. ASL sequence types with single and multiple post-labeling delays (PLDs), for which BIDS could be extended with minimal changes, were also included. Examples include adding a single BIDS-field to indicate a Look-Locker readout or allowing a scalar value in an array format to support the multiple contrast types of Quantitative STAR labeling of arterial regions (QUASAR) ${ }^{15,16}$.

\section{ASL-BIDS structure}

While ASL can provide information relating to functional activation of the brain, it is most commonly used to measure the fundamental physiological parameter perfusion. Perfusion reflects baseline metabolic demand rather than transient neural activity, distinguishing it from the existing BIDS data type 'func'. Therefore, a new perfusion data type 'perf' for ASL-related data was defined, which can also be used for other perfusion-related BIDS extensions in the future, such as dynamic susceptibility contrast (DSC) MRI ${ }^{17}$.

A consensus was reached to store image volumes in the same order as it was acquired. This decision preserves data integrity and allows easy review of any temporal effects or artifacts, such as head motion, functional ASL, or reactivity measurements based on, for example, $\mathrm{CO}_{2}$ inhalation or acetazolamide infusion ${ }^{4}$. Additionally, it provides more flexibility for various multiPLD acquisitions and labeling approaches, such as Look-Locker and QUASAR ${ }^{15,16}$. However, it should be noted that the order in which the DICOM images are exported from the scanner may differ from the acquisition order, in which case the volumes should be sorted back to the acquisition order when storing in ASL-BIDS format. For similar reasons, a consensus was reached to keep any calibration (M0) acquisition coherent with the original acquisition, therefore allowing the 'm0scan' to be part of the ASL time series or stored as a separate file. Although recommended by the 2015 ISMRM Perfusion Study Group ASL recommendations ${ }^{1}$, there are still many studies in which ASL is acquired without an M0 acquisition. Therefore, an 'MOType' field was created that specifies the type of MO information to create flexibility. When a blood M0 value is estimated using a different technique, it is recommended to specify the origin of this estimate in the dataset README file. This specification should include information on the measurement methodology, tissue type of the MO measurements, and conversion strategy to blood M0.

Since ASL relies on fast readout techniques, it is often sensitive to distortion or blurring resulting from $\mathrm{B} 0$ inhomogeneities. Such artifacts can be corrected, for example, by estimation of a B0 field map. Most existing field map approaches in BIDS could already be applied to ASL, except for the often-used reversed phase-encoding polarity (PEpolar) technique. This BIDS extension adds the option to store the 'm0scan' with the PEpolar approach in the 'fmap' directory, following the original BIDS specification for field map images. 


\section{Unit scaling}

MR images are often scaled during reconstruction and exported in 12-bit DICOM files to avoid digitization artifacts. Scale factors may differ between the ASL raw time series and the M0 image, especially when the maximum magnitude varies significantly due to the use of background suppression ${ }^{1}$. Proper restoration of the acquired ASL values is crucial for the absolute quantification of perfusion maps.

In addition to the standard DICOM scale slope tags, further scale factors are either stored in private DICOM fields, specified in a separate sequence parameter file, or need to be requested from the vendor. Traditionally, these scaling factors were applied in the quantification phase of an image processing pipeline. The storage of scaling factors in BIDS would make it unclear if, and at which stage, these factors had been applied. Furthermore, any scaling factors in private fields may be removed during the default anonymization of DICOM files. Therefore, a consensus was reached that it is required to apply all scaling defined in the DICOM file (or other source file types) to the data during conversion to BIDS. This strategy avoids propagating the heterogeneity of (DICOM) scale factors to BIDS. More vendor and sequence-specific details are provided elsewhere:

https://github.com/bids-standard/bids-starter-kit/wiki/Creating-a-BIDS-compatible-ASL-dataset.

\section{ASL-BIDS requirements}

Several BIDS fields were added during the development phase of ASL-BIDS, serving the needs of both clinical users and advanced sequence developers. Therefore, it was decided to rank requirement levels based on their necessity for quantification. 'REQUIRED' fields comprise essential parameters for CBF quantification as defined in the 2015 ISMRM Perfusion Study Group ASL recommendations ${ }^{1}$. Parameters that may improve quantification or explain systematic differences between scanners or ASL sequences are labeled as 'RECOMMENDED'. For example, the 'AcquisitionVoxelSize' is 'recommended' as it can be important to consider for grey matter (GM) mask definition or partial volume correction when the reconstruction resolution is not equal to the acquisition resolution ${ }^{1}$. Other parameters were categorized as 'OPTIONAL', although they can still be recommended in specific cases. For example, certain populations with pathology-dependent labeling efficiency may benefit from calibration by phase-contrast flow quantification ${ }^{18}$. The value can then be provided in the field 'LabelingEfficiency' with additional details on the methodology of the estimation given in the dataset README file.

Several non-required pre-existing BIDS fields were defined as required for ASL. Examples include 'MagneticFieldStrength', which is required to select default values for blood and tissue $\mathrm{T} 1, \mathrm{~T} 2$, and T2*1,4. 'SliceTiming' lists the times that specify the acquisition time of each slice with respect to the start of the volume acquisition. This knowledge is required for calculating the effective post-labeling delay for 2D multi-slice sequences. The 'RepetitionTimePreparation' is required for the 'm0scan' to compensate for incomplete T1 relaxation ${ }^{1,4,19}$. Additionally, 'EchoTime' and 'FlipAngle' are required for quantification, mainly if they differ between the ASL time series and the 'm0scan'. Also, the BIDS field 'TotalAcquiredPairs', in which the exact number of 'control'-'label' pairs is specified, is required as it can be needed to reconstruct the scale slope for GE's product sequence. 


\section{Specification of the labeling plane}

Often, abnormal quantitative perfusion values can be explained by suboptimal positioning of the labeling slab. Specific fields describing the position and orientation of the labeling plane are available both in the NEMA DICOM C.8.13.5.14 MR Arterial Spin Labeling Macro ${ }^{6,7}$ and in ASL-BIDS. While these provide a complete description of the exact position with respect to the field of view, they do not reflect the placement with respect to vascular anatomy when a manual or semi-automatic approach was employed instead of a fixed position. ASL-BIDS thus provides an additional free-text field 'LabelingLocationDescription' and an anonymized screenshot '*_asllabeling.jpg' of the labeling plane planning to better describe the position with respect to the subject's anatomy.

\section{ASL-BIDS resources}

This BIDS extension for ASL has been implemented in the BIDS validator, which can be used to test BIDS compatibility. Additionally, several DICOM to BIDS conversion tools and ASL processing software packages have adopted ASL-BIDS (Table 1). To provide examples of BIDS compliant ASL-datasets, five publicly available ASL datasets in BIDS format are freely accessible at https://github.com/bids-standard/bids-examples (Table 2). Additionally, the Open Source Initiative for Perfusion Imaging (OSIPI) ASL MRI challenge datasets ${ }^{20}$ are released in BIDS format. 


\section{Discussion}

This ASL-BIDS extension faced two main challenges compared to existing BIDS data types. Firstly, considerable variability exists between vendors, scanners, and research labs in the implementation, reconstruction, and export of ASL data. Secondly, ASL measures a quantifiable metric. This characteristic renders the accurate reporting of scale slopes essential for quantification, reproducibility, and comparability of ASL studies.

The main limitation of this BIDS extension is that only the ASL approaches recommended in the 2015 ISMRM Perfusion Study Group ASL recommendations are included ${ }^{1}$. However, advanced ASL approaches such as time-encoded, vessel-encoded, velocity-selective, and diffusion-weighted ASL, as well as functional ASL, may be implemented in a future ASL-BIDS release when their usage has expanded, and a consensus is reached ${ }^{21,22}$. Another essential future extension should be the BIDS definition of ASL image processing derivatives. Users of ASL-BIDS should also be aware that it may not always be possible to derive all required and relevant ASL-BIDS parameters from the header of the primary DICOM image. Therefore, it is essential to check that all BIDS required and relevant fields are present, for which the BIDS validator can be a helpful tool: https://bids-standard.github.io/bids-validator/. Finally, ASL applications are not limited to the brain ${ }^{23}$. Whereas ASL-BIDS could perhaps be used for other body parts, ASL-BIDS is validated in ASL images of the brain only.

Despite the complexities involved, this effort has already managed to achieve a high level of adoption. Several ASL pipeline developers were included in this effort, leading to establishing three major pipelines compatible with ASL-BIDS data so far (Table 1). Additionally, ASL-BIDS was endorsed by the majority of the ASL community (Supplementary Information) and is supported by the COST Action CA18206 - Glioma MR Imaging 2.024 - http://glimr.eu - and the Open Source Initiative for Perfusion Imaging (OSIPI) ${ }^{17}$ - https://www.osipi.org/. Due to this initial buy-in and the growing recognition of the benefits of standardization ${ }^{1}$, it is expected that ASL-BIDS will have a broad uptake in the community. In the future, ASL-BIDS - in conjunction with the OSIPIS'S ASL lexicon ${ }^{25}$ - may encourage NEMA and the MRI vendors to include missing BIDS parameters as DICOM fields.

\section{Take-home messages}

1. ASL-BIDS supports the clinically recommended ASL acquisitions described in the 2015 ISMRM Perfusion Study Group ASL recommendations ${ }^{1}$. Advanced ASL sequences and ASL-BIDS derivatives may be implemented in a future release.

2. Incompleteness and underuse of NEMA DICOM fields ${ }^{6,7}$ complicate ASL-BIDS conversion automation, requiring manual completion of the BIDS fields for ASL.

3. ASL and MO are stored in their acquisition order to preserve data integrity and allow review of temporal effects.

4. ASL-BIDS conversion should be performed shortly after data acquisition and before any anonymization to guarantee proper scaling. 


\section{Methods}

The ASL-BIDS extension was initiated by a steering group of ASL experts following the principles behind the original BIDS specification ${ }^{10}$. A first draft was prepared, which was shared online from May 2017 until May 2020 with the international ASL community for feedback and suggestions. All comments and suggestions were incorporated or included in a discussion agenda, depending on the impact and clarity of the feedback. This draft was refined during several teleconferences and face-to-face meetings with ASL experts, such as from the European ASL COST-action (BM1103 - Arterial Spin Labelling Initiative in Dementia www.cost.eu/actions/BM1103). In March 2019, the draft was presented at the ISMRMendorsed International Workshop on Arterial Spin Labeling MRI: Technical Updates and Clinical Experience, held at the University of Michigan ${ }^{26}$. Final concepts and issues were discussed during successive teleconferences within a smaller working group. Discrepancies were resolved by discussion and voting, allowing finalization of the specification in August 2020.

Simultaneously, five example datasets were made publicly available for ASL sequences of GE, Philips, and Siemens. In addition, the BIDS-validator ${ }^{10}$ was updated and internally validated using 51 non-public clinical datasets from various ASL acquisition techniques. Additionally, efforts were initiated to adapt existing software analysis tools for ASL-BIDS compatibility ${ }^{10,27}$.

From September 2020 until November 2020, the BIDS extension for ASL, including Appendix XII - Arterial Spin Labeling, the example datasets, and the BIDS validator, were disseminated throughout the ASL and BIDS community for testing and endorsement. BIDS version 1.5.0 was released on February 24, 2021, with ASL-BIDS incorporated:

https://bids-specification.readthedocs.io/en/v1.5.0/. 


\section{Acknowledgments}

We thank all researchers within the ASL and BIDS community who were involved in the developmental process of ASL-BIDS for providing advice and feedback. TO was supported by a Sir Henry Dale Fellowship jointly funded by the Wellcome Trust and the Royal Society (Grant Number 220204/Z/20/Z). The Wellcome Centre for Integrative Neuroimaging is supported by core funding from the Wellcome Trust (203139/Z/16/Z). DT was supported by the UCL Leonard Wolfson Experimental Neurology Centre (PR/ylr/18575), UCLH NIHR Biomedical Research Centre, and the Wellcome Trust (Centre award 539208). The BIDS maintainers CM, RB, and FF were supported by the National Institute Of Mental Health of the National Institutes of Health under Award Number R24MH117179. HM is supported by the Dutch Heart Foundation (2020T049), by the Eurostars-2 joint program with co-funding from the European Union Horizon 2020 research and innovation program, provided by the Netherlands Enterprise Agency (RvO), and by the EU Joint Program for Neurodegenerative Disease Research, provided by the Netherlands Organisation for health Research and Development and Alzheimer Nederland.

\section{Author contributions}

PC Daily management, moderating communications, development specification, providing example dataset, testing example datasets, validator testing, implementation in existing BIDS, manuscript writing.

MC Daily management, development specification, update BIDS validator, specification and validator testing, implementation in existing BIDS, critical review, and final approval of the manuscript submitted.

TO Development specification, critical review, and final approval of the manuscript submitted. DT Providing example datasets, critical review specification, critical review, and final approval of the manuscript submitted.

PV Development specification, critical review, and final approval of the manuscript submitted.

SE Development specification, critical review, and final approval of the manuscript submitted. AOT Development specification, validator testing, critical review, and final approval of the manuscript submitted.

TK Development specification, critical review, and final approval of the manuscript submitted.

JW Development specification, providing example dataset, critical review, and final approval of the manuscript submitted.

SV Providing example dataset, critical review specification, critical review, and final approval of the manuscript submitted.

JK Critical review specification, validator testing, critical review, and final approval of the manuscript submitted.

EA Providing example dataset, critical review specification, critical review, and final approval of the manuscript submitted.

MO Development specification, critical review specification, validator testing, critical review, and final approval of the manuscript submitted.

the BIDS maintainers Support update validator, implementation in existing BIDS, critical review specification, critical review, and final approval of the manuscript submitted.

JD Validator testing, critical review specification, critical review, and final approval of the manuscript submitted. 
HL Critical review specification, critical review, and final approval of the manuscript submitted. DA Critical review specification, critical review, and final approval of the manuscript submitted. MAC Initiation, development specification, providing example dataset, critical review, and final approval of the manuscript submitted.

LH Development specification, critical review, and final approval of the manuscript submitted. JP Daily management, development specification, preparation of example datasets, implementation in existing BIDS, manuscript writing.

HM Initiation, daily management, moderating communications, development specification, implementation in existing BIDS, manuscript writing.

\section{Consortium}

The BIDS maintainers:

Stefan Appelhoff ${ }^{23}$, Ross Blair ${ }^{24}$, Franklin Feingold ${ }^{24}$, Rémi Gau ${ }^{25}$, Christopher J Markiewicz ${ }^{24}$, Taylor Salo 26

23. Center for Adaptive Rationality, Max Planck Institute for Human Development, Lentzeallee 94, Berlin, Berlin, 14195, Germany;

24. Department of Psychology, Stanford University, 450 Jane Stanford Way, Stanford, CA, 94305, USA;

25. Institute of psychology, Université Catholique de Louvain, Louvain la neuve, MD, Belgium;

26. Department of Psychology, Florida International University, 11200 SW 8th Street, Miami, FL, 33199, USA

\section{Competing interests}

The authors declare that they have no conflict of interest. 


\section{Tables}

Table 1 - Overview of tools and software packages compliant with ASL-BIDS.

\begin{tabular}{|c|c|c|}
\hline Tool/Software & Description & Available at \\
\hline \multicolumn{3}{|l|}{ BIDS-validator } \\
\hline $\begin{array}{l}{ }^{*} \text { BIDS- } \\
\text { validator }{ }^{10,27}\end{array}$ & $\begin{array}{l}\text { Validator provided by the BIDS } \\
\text { standard, evaluating the compliance of } \\
\text { the BIDS-converted dataset with the } \\
\text { standard, including metadata and } \\
\text { conflicts between data reported in the } \\
\text { JSON file, compared to the data } \\
\text { recorded in the NIfTI header. }\end{array}$ & https://github.com/bids-standard/bids-validator \\
\hline \multicolumn{3}{|c|}{ DICOM to BIDS conversion tools } \\
\hline BIDScoin & $\begin{array}{l}\text { A user-friendly, open-source python } \\
\text { toolbox. Raw images can easily be } \\
\text { converted to BIDS-compliant datasets } \\
\text { using the Graphical User Interface. }\end{array}$ & https://bidscoin.readthedocs.io/en/stable/ \\
\hline dcm2bids & $\begin{array}{l}\text { A community-centered project, } \\
\text { providing a tool for effortless conversion } \\
\text { of DICOM images to BIDS format. }\end{array}$ & https://pypi.org/project/dcm2bids/ \\
\hline dcm2niix ${ }^{28}$ & $\begin{array}{l}\text { Tool for the conversion of images from } \\
\text { the DICOM format to the NIfTI format. }\end{array}$ & https://github.com/rordenlab/dcm2niix \\
\hline heudiconv & $\begin{array}{l}\text { A flexible DICOM converter, organizing } \\
\text { imaging data into structured directories. } \\
\text { Heudiconv assists in the conversion into } \\
\text { BIDS format. }\end{array}$ & https://heudiconv.readthedocs.io/en/latest/ \\
\hline pyBIDSconv & $\begin{array}{l}\text { A Graphical User Interface tool to } \\
\text { convert MRI DICOMs into BIDS format. }\end{array}$ & https://github.com/DrMichaelLindner/pyBIDSconv \\
\hline
\end{tabular}

\section{ASL processing software packages}

\begin{tabular}{|c|c|c|}
\hline ASLDRO 29 & $\begin{array}{l}\text { Open-source tool to generate BIDS- } \\
\text { compliant simulated ASL digital } \\
\text { reference object data. Raw ASL time } \\
\text { series comprising control, label, and M0 } \\
\text { volumes are synthesized from ground } \\
\text { truth maps according to configurable } \\
\text { acquisition and labeling parameters. }\end{array}$ & https://github.com/gold-standard-phantoms/asldro \\
\hline ASLPrep ${ }^{30}$ & $\begin{array}{l}\text { ASL data preprocessing and cerebral } \\
\text { blood flow computation pipeline, } \\
\text { designed for easy accessibility, state-of- } \\
\text { the-art interface, and robustness to } \\
\text { acquisition variations. }\end{array}$ & https://as/prep.readthedocs.io/en/latest/index.html \\
\hline BASIL $^{31}$ & $\begin{array}{l}\text { Toolbox within the FMRIB Software } \\
\text { Library providing the tools to analyze } \\
\text { ASL datasets with quantification based } \\
\text { on Bayesian inference principles. The } \\
\text { toolbox accepts both single- and multi- } \\
\text { PLD ASL data in BIDS format. }\end{array}$ & https://asl-docs.readthedocs.io/en/latest/ \\
\hline
\end{tabular}


ExploreASL, an SPM-based toolbox for processing, statistical analysis, and quality control of ASL datasets, free for

http://exploreasl.org;

https://github.com/ExploreASL/ExploreASL non-commercial usage.

${ }^{*}=$ extensively tested with 51 non-public clinical datasets from a variety of ASL techniques Abbreviations: BIDS = Brain Imaging Data Structure; JSON = JavaScript Object Notation; NIfTI = Neuroimaging Informatics Technology Initiative; DICOM = Digital Imaging and Communications in Medicine; PLD = post-labeling delay, SPM = Statistical parametric mapping 
Table 2 - Overview of five freely accessible ASL datasets in BIDS format, available for download at https://github.com/bids-standard/bids-examples. All datasets contain a 3D T1W structural scan.

\begin{tabular}{ll} 
Name & Dataset description \\
\hline asI001 & $\begin{array}{l}\text { One volunteer scanned on a GE MR750 3T, using the GE product sequence: a single-PLD PCASL } \\
\text { sequence with segmented stack-of-spirals 3D readout and four background suppression pulses. } \\
\text { ASL time series consists only of the volumes' deltam' and 'm0scan'. }\end{array}$ \\
\hline asI002 & $\begin{array}{l}\text { One volunteer scanned on a Philips Achieva 3T, using the Philips WIP sequence: a single-PLD } \\
\text { PCASL sequence with single-shot 2D-EPI and two background suppression pulses, 'm0scan' } \\
\text { acquired separately. }\end{array}$ \\
\hline asI003 & $\begin{array}{l}\text { One volunteer scanned on a Siemens Trio 3T, using the Siemens C2P (Bremen) sequence: a multi- } \\
\text { PLD PASL sequence with a segmented 3D GRASE readout and two background suppression } \\
\text { pulses, 'm0scan' acquired separately. }\end{array}$
\end{tabular}

asI004 32,33 One volunteer scanned on a Siemens Prisma 3T, using a custom multi-PLD PCASL sequence with a 2D-EPI readout, two background suppression pulses, and 'm0scan' included in the time series. The dataset includes an additional 'm0scan' with reversed phase-encoding direction (PEpolar).

asl005 One volunteer scanned on a Siemens Prisma 3T, using the Siemens WIP sequence: a single-PLD PCASL sequence with segmented 3D GRASE readout and four background suppression pulses, 'm0scan' included in the time series.

Abbreviations: PLD = post-labeling delay, PCASL = pseudo-continuous arterial spin labeling, GRASE = Gradientand spin-echo, PASL = pulse arterial spin labeling, EPI = echo planar, PEpolar = phase-encoding polarity, WIP = work in progress. 


\section{References}

1. Alsop, D. C. et al. Recommended implementation of arterial spin-labeled perfusion MRI for clinical applications: A consensus of the ISMRM perfusion study group and the European consortium for ASL in dementia. Magn Reson Med 73, 102-16 (2015).

2. Almeida, J. R. C. et al. Test-retest reliability of cerebral blood flow in healthy individuals using arterial spin labeling: Findings from the EMBARC study. Magn. Reson. Imaging 45, 2633 (2018).

3. Mutsaerts, H. J. M. M. et al. Inter-Vendor Reproducibility of Pseudo-Continuous Arterial Spin Labeling at 3 Tesla. PLoS ONE 9, (2014).

4. $\quad$ Mutsaerts, H. J. M. M. et al. ExploreASL: An image processing pipeline for multi-center ASL perfusion MRI studies. Neurolmage 219, 117031 (2020).

5. Fedorov, A. et al. DICOM for quantitative imaging biomarker development: a standards based approach to sharing clinical data and structured PET/CT analysis results in head and neck cancer research. PeerJ 4, e2057 (2016).

6. Bidgood, W. D. \& Horii, S. C. Introduction to the ACR-NEMA DICOM standard. Radiogr. Rev. Publ. Radiol. Soc. N. Am. Inc 12, 345-355 (1992).

7. ACR-NEMA. DICOM PS3.3 2018d - Information Object Definitions - C.8.13.5.14 MR Arterial Spin Labeling Macro (Current). http://dicom.nema.org/medical/Dicom/2018d/output/chtml/part03/sect_C.8.13.5.14.html (2021).

8. Dolui, S. et al. The Open Source Initiative for Perfusion Imaging (OSIPI): ASL Pipeline inventory. in Proceedings of the Annual Meeting of ISMRM (2021).

9. Cox, R. et al. A (sort of) new image data format standard: NiFTI-1. in 10th Annual Meeting of the Organization for Human Brain Mapping. (2004).

10. Gorgolewski, K. J. et al. The brain imaging data structure, a format for organizing and describing outputs of neuroimaging experiments. Sci. Data 3, 160044 (2016).

11. Niso, G. et al. MEG-BIDS, the brain imaging data structure extended to magnetoencephalography. Sci. Data 5, 180110 (2018).

12. Pernet, C. R. et al. EEG-BIDS, an extension to the brain imaging data structure for electroencephalography. Sci. Data 6, 103 (2019).

13. Holdgraf, C. et al. iEEG-BIDS, extending the Brain Imaging Data Structure specification to human intracranial electrophysiology. Sci. Data 6, 102 (2019).

14. Brain Imaging Data Structure. Positron Emission Tomography. Brain Imaging Data Structure https://bids-specification.readthedocs.io/en/stable/04-modality-specific-files/09positron-emission-tomography.html (2021).

15. Günther M, Bock M, \& Schad Lr. Arterial spin labeling in combination with a look-locker sampling strategy: inflow turbo-sampling EPI-FAIR (ITS-FAIR). Magnetic resonance in medicine vol. 46 https://pubmed.ncbi.nlm.nih.gov/11675650/ (2001).

16. Chappell, M. A., Woolrich, M. W., Petersen, E. T., Golay, X. \& Payne, S. J. Comparing model-based and model-free analysis methods for QUASAR arterial spin labeling perfusion quantification. Magn. Reson. Med. 69, 1466-1475 (2013).

17. OSIPI. Open Source Initiative for Perfusion Imaging. https://www.osipi.org/ (2021).

18. Aslan, S. et al. Estimation of Labeling Efficiency in Pseudocontinuous Arterial Spin Labeling. Magn. Reson. Med. 63, 765-771 (2010).

19. Pinto, J. et al. Calibration of arterial spin labeling data-potential pitfalls in postprocessing. Magn. Reson. Med. 83, 1222-1234 (2020).

20. Anazodo, U. C. et al. The Open Source Initiative for Perfusion Imaging (OSIPI) ASL MRI Challenge. in Proceedings of the Annual Meeting of ISMRM (2021).

21. Steketee, R. M. E. et al. Quantitative Functional Arterial Spin Labeling (fASL) MRI Sensitivity and Reproducibility of Regional CBF Changes Using Pseudo-Continuous ASL Product Sequences. PLOS ONE 10, e0132929 (2015).

22. van Osch, M. J. et al. Advances in arterial spin labelling MRI methods for measuring perfusion and collateral flow. J. Cereb. Blood Flow Metab. 38, 1461-1480 (2018). 
23. Nery, F. et al. Consensus-based technical recommendations for clinical translation of renal ASL MRI. Magn. Reson. Mater. Phys. Biol. Med. 33, 141-161 (2020).

24. Clement, P. et al. GliMR: Cross-Border Collaborations to Promote Advanced MRI Biomarkers for Glioma. J. Med. Biol. Eng. 1-11 (2020) doi:10.1007/s40846-020-00582-z.

25. OSIPI Task Force 4.1. Arterial Spin Labeling perfusion imaging and analysis lexicon and reporting recommendations (v0.1). https://docs.google.com/document/d/e/2PACX1vQPhnYciWScZ2vqjzBWUO72gXKKD2UA95q081OA7-

2dnLiy9kOimXq1axA7E7xRxbZOmJAb9vMDZ_-D/pub (2021).

26. Clement, P. et al. ASL-BIDS, the brain imaging data structure extension for arterial spin labeling. in Magn. Reson. Mat. Phys. Biol. Med. vol. 32(1 Supplement) s147-s148 (2019).

27. Gorgolewski, K. J. et al. BIDS apps: Improving ease of use, accessibility, and reproducibility of neuroimaging data analysis methods. PLoS Comput. Biol. 13, e1005209 (2017).

28. Li, X., Morgan, P. S., Ashburner, J., Smith, J. \& Rorden, C. The first step for neuroimaging data analysis: DICOM to NIfTI conversion. J. Neurosci. Methods 264, 47-56 (2016).

29. Oliver-Taylor, A. et al. ASLDRO: Digital reference object software for Arterial Spin Labelling. in Proceedings of Annual Meeting of ISMRM 2731 (2021).

30. Adebimpe, A. et al. ASLPrep: A Generalizable Platform for Processing of Arterial Spin Labeled MRI and Quantification of Regional Brain Perfusion. bioRxiv 2021.05.20.444998 (2021) doi:10.1101/2021.05.20.444998.

31. Chappell, M. A., Groves, A. R., Whitcher, B. \& Woolrich, M. W. Variational Bayesian Inference for a Nonlinear Forward Model. IEEE Trans. Signal Process. 57, 223-236 (2009).

32. Okell, T. W., Chappell, M. A., Kelly, M. E. \& Jezzard, P. Cerebral blood flow quantification using vessel-encoded arterial spin labeling. J. Cereb. Blood Flow Metab. 33, 1716-1724 (2013).

33. Woods, J. G., Chappell, M. A. \& Okell, T. W. A general framework for optimizing arterial spin labeling MRI experiments. Magn. Reson. Med. 81, 2474-2488 (2019). 